\title{
Tabanidae (Diptera) do estado do Maranhão, Brasil. III. Descrição de Philipotabanus (Mimotabanus) henriquesi sp. nov.
}

\author{
Francisco Limeira-de-Oliveira ${ }^{1}$, Inocêncio de Sousa Gorayeb ${ }^{2} \&$ José Albertino Rafael $^{3}$
}

\begin{abstract}
${ }^{1}$ Laboratório de Estudos dos Invertebrados, Universidade Estadual do Maranhão, 65604-380 Caxias-MA, Brasil. franciscolimeira@cesc.uema.br ${ }^{2}$ Museu Paraense Emílio Goeldi, 66040-170 Belém-PA, Brasil. gorayeb@museu-goeldi.br

${ }^{3}$ Instituto Nacional de Pesquisas da Amazônia, Caixa Postal 478, 69011-970 Manaus-AM, Brasil. jarafael@inpa.gov.br
\end{abstract}

\begin{abstract}
Tabanidae (Diptera) from the state of Maranhão, Brazil. III. Description of Philipotabanus (Mimotabanus) henriquesi sp. nov. Philipotabanus (Mimotabanus) henriquesi, sp. nov., is described and illustrated based on 30 females and two males specimens collected in open vegetation of "Cerrado", in Carolina, Alto Parnaíba and Mirador municipalities, in southern Maranhão state, Brazil. This is the first record of species of the subgenus in Brazil.
\end{abstract}

KEYWORDS. Biodiversity; Brazil; horsefly, taxonomy.

RESUMO. Tabanidae (Diptera) do estado do Maranhão, Brasil. III. Descrição de Philipotabanus (Mimotabanus) henriquesi sp. nov. Philipotabanus (Mimotabanus) henriquesi sp. nov. é descrita e ilustrada baseada em 30 fêmeas e dois machos coletados em vegetação de Cerrado, nos municípios de Carolina, Alto Parnaíba e Mirador, região sul do estado do Maranhão, Brasil. Este é o primeiro registro de uma espécie do subgênero no Brasil.

PALAVRAS-CHAVE. Biodiversidade; Brasil; mutucas; taxonomia.

Fairchild \& Burger (1994) catalogaram 25 espécies em Philipotabanus Fairchild, 1943, sendo nove em $P$. (Mimotabanus) Fairchild, 1964, registradas no Panamá, Colômbia e Peru. A maioria das espécies neste subgênero foi descrita por Fairchild (1964) e o gênero foi discutido por Henriques (2006) que acrescentou mais uma espécie ao gênero e apresentou uma chave dicotômica para as espécies registradas na Amazônia.

Um lote de espécimes coletados em vegetação de Cerrado do sul do estado do Maranhão, Brasil, segue para a dicotomia de $P$. (Mimotabanus) na chave de Coscarón \& Papavero (1993) e em face da distribuição geográfica disjunta e atributos diferentes das espécies já descritas, está sendo descrita como espécie nova, a décima ao subgênero.

A descrição utiliza a terminologia de McAlpine (1981). O material está depositado nas seguintes instituições: Coleção Zoológica do Maranhão da Universidade Estadual do Maranhão em Caxias, Maranhão, Brasil (CZMA); Museu Paraense Emílio Goeldi, Belém, Pará, Brasil (MPEG); Instituto Nacional de Pesquisas da Amazônia, Manaus, Amazonas, Brasil (INPA); Museu de Zoologia da Universidade de São Paulo, São Paulo, Brasil (MZUSP) e no Museu Nacional do Rio de Janeiro, Rio de Janeiro, Brasil (MNRJ). No material examinado foi utilizado as seguintes abreviaturas: F (fêmea), M (macho).

\section{Philipotabanus (Mimotabanus) henriquesi, sp. nov.} (Figs. 1-16)

Diagnose. Espécimes pequenos, média de $9 \mathrm{~mm}$ de comprimento, predominantemente marrom-claros. Fronte estreita, de lados quase paralelos. Calo frontal grande, marrom, mais estreito do que a largura da fronte, estendendo-se além da sua metade. Mesonoto com faixas submedianas longitudinais de pruína cinza mais distinta na região anterior. Asa com duas manchas transversais escuras, uma basal e outra mediana. Abdômen marrom-claro, dorsalmente com um triângulo maior de cerdas brancas na margem posterior do tergito 4 e um menor no tergito 5; póstero-lateralmente com cerdas brancas nos tergitos $1-3$.

Holótipo fêmea. Corpo (Fig. 1) com 9,35 mm de comprimento. Olhos marrom-avermelhados com cerdas diminutas, esparsas, visíveis a grande aumento. Fronte (Fig. 3) relativamente estreita, de lados subparalelos, ligeiramente convergentes abaixo, com pruinosidade cinza-escura e cerdas amarelas, exceto pelo vértice com pruinosidade cinza-clara e cerdas pretas. Medidas da fronte: altura $1,56 \mathrm{~mm}$; largura na base $0,31 \mathrm{~mm}$; largura no vértice $0,31 \mathrm{~mm}$; índice frontal 5,03; índice de divergência 1,00. Calo frontal grande, em forma de gota, marrom, liso, brilhante, mais estreito do que a fronte, estendendo-se além da metade da fronte. Ocelos indistintos. Subcalo com pruinosidade cinzaescura densa, tendendo ao amarelo em diferentes ângulos de incidência da luz. Antena (Figs. 2, 4) com escapo e pedicelo amarelos, o primeiro com cerdas pretas e amarelas mescladas, o segundo com cerdas pretas. Escapo cerca de duas vezes o comprimento do pedicelo. Flagelo amarelo-laranja com estilo marrom e cerca de metade do comprimento da placa basal. Frontoclípeo marrom com pruína cinza, levemente amarelada, com cerdas marrom-claras nas proximidades da base da antena. Parafasciália marrom com pruína cinza, levemente mais clara do que o subcalo e frontoclípeo. Gena e pós-gena pretas com pruína cinza e cerdas marrom-claras. Palpo (Figs. 2, 5) marromclaro; primeiro segmento cilíndrico com cerdas pretas delgadas; 


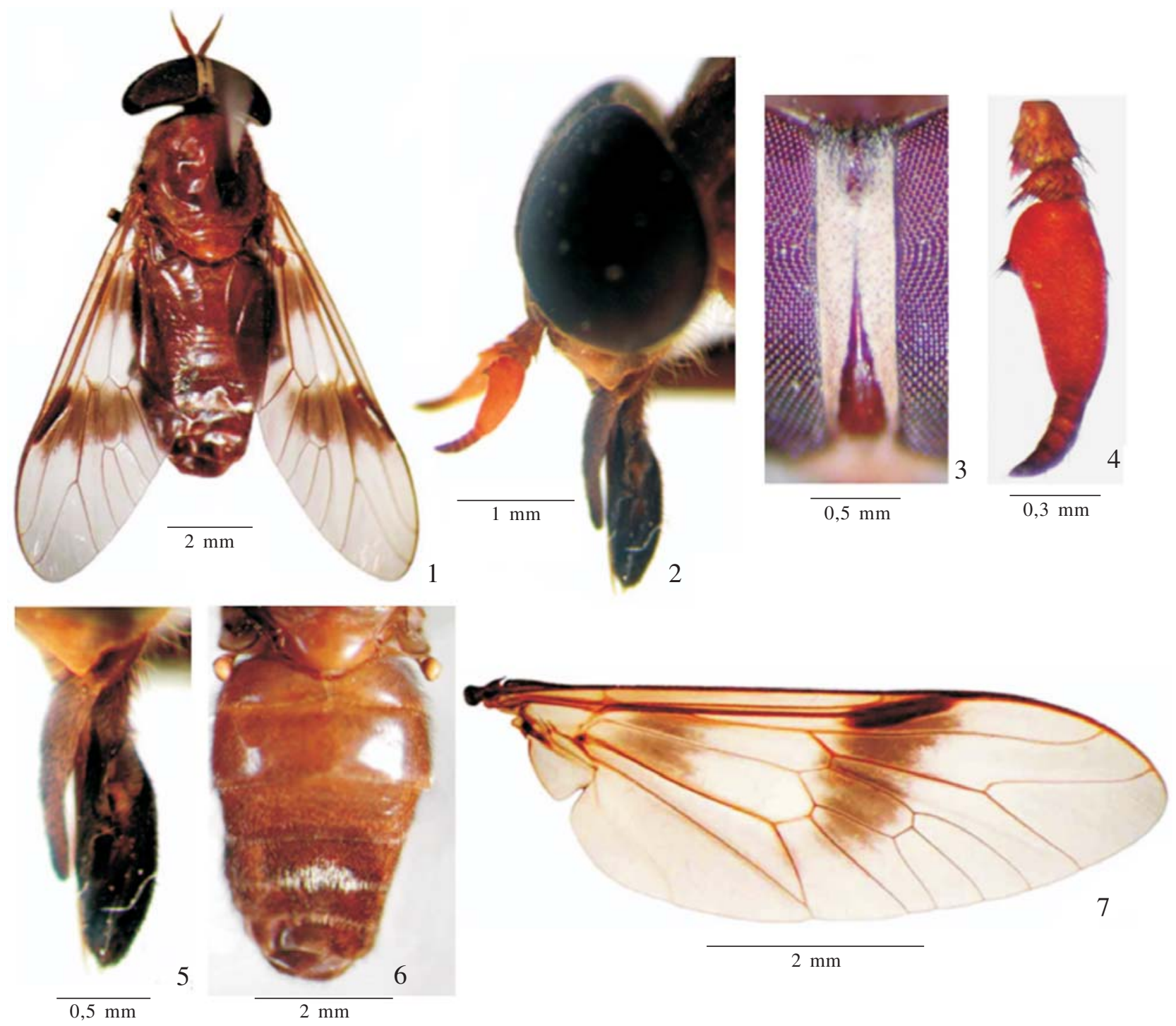

Figs. 1-7. Philipotabanus (Mimotabanus) henriquesi, sp. nov., fêmea. 1, espécime em vista dorsal; 2, cabeça, vista lateral; 3, fronte; 4, antena; 5, palpo e probóscide; 6, abdomen; 7, asa.

segundo segmento mais comprido do que o flagelo, um tanto achatado lateralmente, mais largo na base em vista lateral (inflado), com pruinosidade cinza e cerdas pretas curtas. Probóscide (Figs. 2, 5) de comprimento aproximado ao da altura da cabeça, marrom, opaca, exceto na base, ventralmente, marrom sub-brilhante com cerdas marrons delgadas e pretas curtas mescladas. Labela grande e membranosa com cerdas escuras. Occipício com pruína cinza e cerdas amarelo-claras. Tórax com escudo marrom e uma faixa longitudinal mediana, intra cerdas dorsocentrais, marrom-clara que escurece em direção ao escutelo; essa faixa, em vista dorsal, margeada anterolateralmente por uma faixa de pruinosidade cinza conspícua até a altura da sutura transversa, onde se torna evanescente. Mesonoto com cerdas brancas a amarelo-claras e pretas mescladas. Lobo pós-pronotal amarelo, com fina pruinosidade cinza e cerdas brancas. Notopleura amarela, a metade dorsal com cerdas brancas, a metade ventral com cerdas pretas. Lobo pós-alar amarelo com cerdas brancas. Escutelo amarelo, com pruína cinza esparsa e cerdas brancas. Proepisterno com pruína cinza distinta e cerdas brancas delgadas. Proepímero amarelo com pruína cinza esparsa e cerdas marrom-claras. Mesopleura marrom, exceto anepímero amarelo, todos com pruinosidade cinza mais distinta nos escleritos mais escuros; anepisterno com cerdas amarelodouradas a marrons; catepisterno e anepímero com cerdas brancas. Catatergito marrom com pruína cinza e tufo distinto de cerdas brancas delgadas. Pernas marrons com cerdas predominantemente pretas; a perna anterior levemente mais escura que as demais. Asa (Fig. 7): comprimento 9,19 mm, largura 3,23 mm. Tégula marrom-escura a preta. Basicosta amarela, com poucas cerdas pretas, contrastando com a costa adjacente com muitas cerdas pretas. Pterostigma e veias 

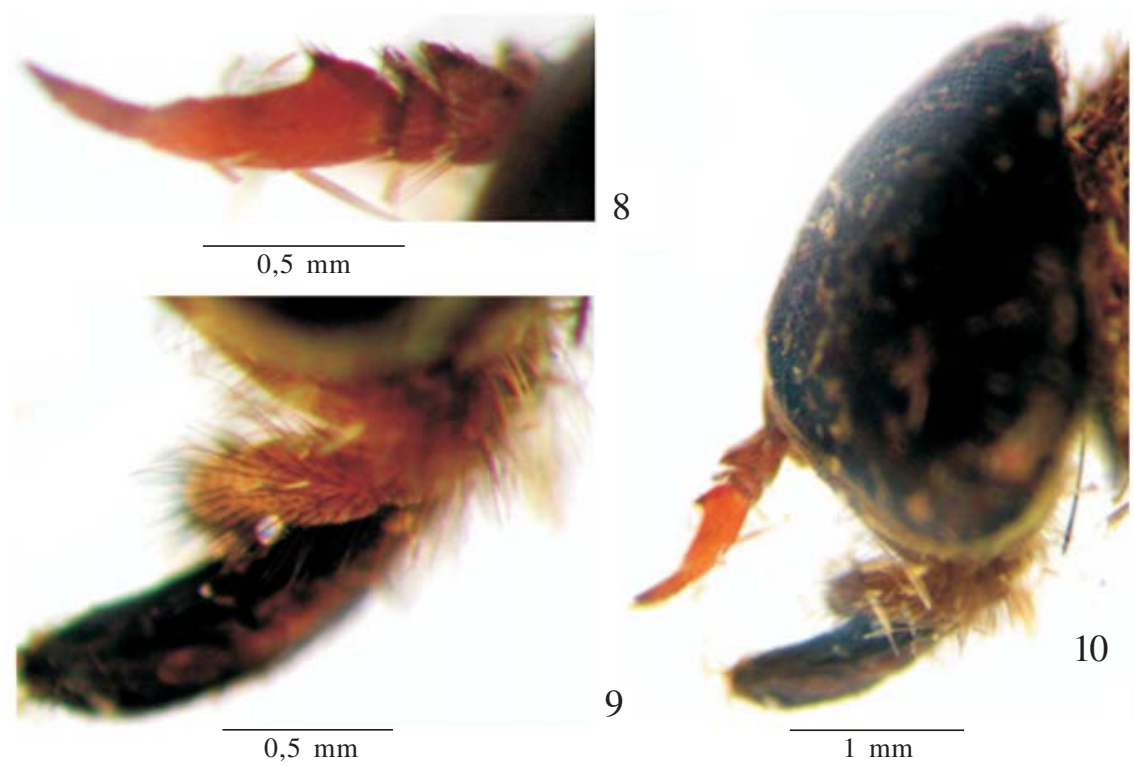

Figs. 8-10. Philipotabanus (Mimotabanus) henriquesi, sp. nov., macho. 8, antena; 9, palpo e probóscide; 10, cabeça, vista lateral.

marrons. Asa hialina com duas manchas transversais, uma basal e outra mediana (Fig. 7). Célula $\mathrm{r}_{1}$ com mancha marromescura abaixo do pterostigma. Caliptras amarelo-claras com franja de cerdas amarelas. Halter predominantemente amarelo. Abdômen (Fig. 6) marrom-claro, os últimos dois tergitos e últimos três esternitos levemente mais escuros. Tergitos com cerdas predominantemente pretas, exceto por um triângulo maior de cerdas brancas no tergito 4 e um menor no tergito 5 ; póstero-lateralmente com cerdas brancas nos tergitos 1-3. Terminália como nas Figuras 11-14. Cerco subcircular (Fig. 12). Cápsula da espermateca alongada (Fig. 13).

Macho (Figs. 8-10) dois espécimes examinados de comprimento médio $11,05 \mathrm{~mm}(10,00-12,10 \mathrm{~mm})$, comprimento médio da asa 10,64 $\mathrm{mm}(9,19-12,10 \mathrm{~mm})$, largura média da asa $3,30 \mathrm{~mm}(3,06-3,55 \mathrm{~mm})$. Olhos com cerdas claras mais longas e densas que na fêmea. Área de omatídios menores marrom, de omatídios maiores, marrom-clara. Probóscide com cerdas da face inferior claras. Antena (Figs. 8, 10) com escapo e pedicelo amarelos; flagelo predominantemente amarelo-laranja, levemente mais escuro no ápice. Palpo como nas Figs. 9-10. Mesonoto com cerdas eretas e mais longas que na fêmea. Proepímero com cerdas claras, anepisterno com cerdas claras na metade anterior e cerdas escuras na metade posterior. Asa igual a da fêmea, porém com cerdas da tégula amarelas. Terminália como nas Figuras 15-16. Gonóstilo (Fig. 15) com pequena protuberância subapical.

Material tipo. Holótipo fêmea: BRASIL, Maranhão, Carolina, Balneário Urupuxete, $07^{\circ} 23^{\prime} 54^{\prime \prime} \mathrm{S}-4^{\circ} 15^{\prime} 00^{\prime \prime} \mathrm{W}$, isca eqüina, 10 13.xii.2001, J. A. Rafael, F. L. Oliveira \& J. F. Vidal (INPA).

Parátipos: mesmos dados do holótipo (1F, INPA); Idem, 0809.i.2008, F. Limeira-de-Oliveira (7F, CZMA); Idem, 28-31.x.2008, F. Limeira-de-Oliveira \& P. A. M. Moraes (5F, INPA). Alto Parnaíba, Balneário Brejo do Campo, armadilha de Malaise, 13-15.x.2004, F. Limeira-de-Oliveira (1F, INPA; 1F, 1M, CZMA; 3F, MPEG); Idem,
Povoado Estiva, $02^{\circ} 45^{\prime}$ S - 44²0'W; atração humana; iv.1989, M. S. Brígida (3F, MPEG). Mirador, Parque Estadual do Mirador, Base da Geraldina, armadilha suspensa, 02-15.x.2006, F. Limeira-de-Oliveira (1F, CZMA; 1F, MNRJ); Idem, 09-16.xi.2006 (1F, MNRJ; 1F, MPEG); Idem, armadilha de Malaise, 15-26.x.2006 (1F, CZMA; 2F, MZUSP); Idem, 01-15.xi.2006 (1F, INPA); Idem, 15-30.xi.2006 (1M, MPEG).

Registros geográficos: Brasil, Maranhão (Carolina, Alto Parnaíba e Mirador).

Variações. Fêmea: comprimento do corpo com média de $9,08 \mathrm{~mm}$ (série típica variando de 8,6-10,32 mm). Fronte: altura média da série típica $1,57 \mathrm{~mm}(1,41-1,72 \mathrm{~mm})$; largura média na base $0,30 \mathrm{~mm}(0,28-0,34 \mathrm{~mm})$; largura média no vértice $0,34 \mathrm{~mm}$ (0,31-0,37 mm); índice frontal médio 5,23 (4,74-6,14); índice de divergência médio $1,13(1,00-1,32)$. Asa com comprimento médio da série típica $9,01 \mathrm{~mm}(8,23-9,68 \mathrm{~mm})$; largura média $3,07 \mathrm{~mm}(2,74-3,39 \mathrm{~mm})$.

Discussão taxonômica. As afinidades entre os gêneros de Tabanidae ainda não estão resolvidas e a monofilia de Philipotabanus não é segura. O gênero é caracterizado, segundo Coscarón \& Papavero (1993) e Henriques (2006), pela presença de cerdas na basicosta, tíbias não infladas, face e palpo pruinosos, protuberância da placa basal do flagelo formando um ângulo obtuso e fronte muito estreita. As características utilizadas para separar os três subgêneros foram apresentadas por Henriques (2006). P. henriquesi, sp. nov., têm as características apresentadas na chave de Coscarón \& Papavero (1993) e Henriques (2006) para esse subgênero. Diferencia-se das demais espécies, além da distribuição geográfica disjunta, já que as espécies descritas anteriormente foram registradas para o Panamá, Colômbia e Peru e esta para o nordeste brasileiro, pela placa basal mais estreita (comparar figura 4 com a figura 92k de Coscarón \& Papavero 1993). Na chave apresentada por Henriques (2006), P. henriquesi sp. nov. segue para a dicotomia 9 de $P$. (M.) opimus Fairchild, 1975 

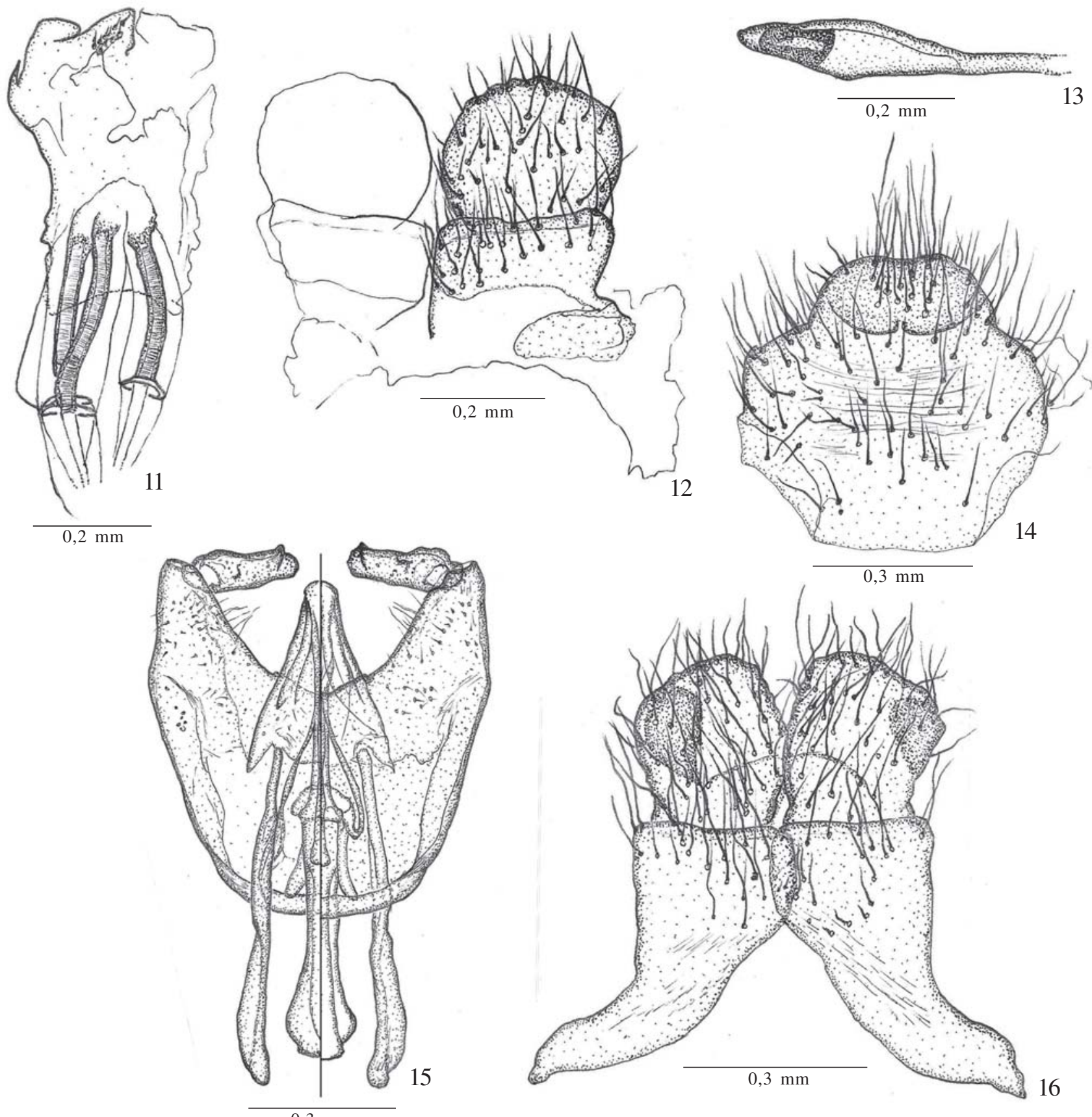

Figs. 11-16. Terminália de Philipotabanus (Mimotabanus) henriquesi, sp. nov. Figs. 11-14, parátipo fêmea: 11) furca genital e base dos dutos da espermateca; 12) tergitos $9-10$ e cercos; 13) cápsula da espermateca; 14) esternito 8 e gonapófises. Figs. 15-16, parátipo macho: 15) edeago e gonóstilos; 16) epândrio e cercos.

pelo tamanho e índice frontal muito próximos. De acordo com Fairchild (1975), P. opimus tem coloração muito variável onde o calo frontal pode ser amarelo, marrom ou preto, a mancha no meio da asa pode ser quase preta a indistinta e o abdômen pode ter triângulos medianos ou bandas completas na margem posterior. Por essas características não é possível diferenciála de $P$. henriquesi sp. nov. No entanto, Henriques (2006) complementa os atributos de $P$. opimus citando a presença de triângulos medianos prateados na margem posterior dos tergitos $2-4$ e a basicosta glabra. Por essas características é possível diferenciar $P$. henriquesi sp. nov. que têm triângulos de cerdas brancas na margem posterior dos tergitos 4 e 5 e basicosta com poucas cerdas.

Devido à dificuldade de obtenção de empréstimo de espécimes de coleções estrangeiras, P. (M.) henriquesi, sp. nov. foi comparada com atributos das descrições originais das demais espécies que os diferenciam da descrição apresentada acima. 
P. (M.) annectans Fairchild, 1975 possui mesonoto preto sub-brilhante, asa com uma mancha que se estende do pterostigma à célula discal e tergitos pretos. $P$. (M.) fucosus Fairchild, 1958 possui placa basal da antena marrom-escura avermelhada na base e escura no ápice, mesonoto marromavermelhado, asa com uma mancha indo do pterostigma à célula anal, tergitos marrom-escuros. $P$. (M.) inauratus (Fairchild, 1947) possui antena, palpo e mesonoto pretos, asa predominantemente preta com ápice hialino, tergitos pretos. $P$. (M.) opimus, é a espécie que cromaticamente se aproxima mais de $P$. henriquesi, $\mathbf{s p . ~ n o v . ~ e ~ d i f e r e n c i a - s e ~ p e l o s ~ a t r i b u t o s ~}$ apresentados acima. P. (M.) phalaropygus Fairchild, 1964 possui palpo, mesonoto, pernas e abdômen pretos. $P$. (M.) plenus (Hine, 1907) possui aparência de um muscóideo, com faixas longitudinais distintas no mesonoto, asa hialina, exceto pterostigma escuro e tergitos com pruína azulada. $P$. (M.) porteri Fairchild, 1975 possui placa basal escura, tergitos $1 \mathrm{e}$ 2 com pruína cinza, demais tergitos pretos. P. (M.) tanypterus Wilkerson, 1979 possui asa uniformemente enfuscada, sem manchas. P. (M.) vulpinus Fairchild, 1975 possui o flagelo da antena marrom-avermelhado, mesonoto marrom-escuro a preto e pernas e tergitos pretos.

Ambiente e sazonalidade. Os espécimes foram coletados em mata ciliar de cerrado, nos meses de abril, outubro-janeiro.

Etimologia. O nome da espécie homenageia o entomólogo Dr. Augusto Loureiro Henriques do INPA, que vem contribuindo com as coleções e incrementando o conhecimento dos Tabanidae neotropicais.
Agradecimentos. Ao Márcio Cutrim M. de Castro pela edição das fotos; à Agroindustrial Serra Grande (AGRO SERRA) e à Cooperativa do Parque Estadual do Mirador (COOPERMIRA) pelo apoio logístico quando da realização das pesquisas de campo. Este estudo é parte da tese de doutorado do autor sênior apresentada ao Curso de Pós-graduação em Entomologia, Instituto Nacional de Pesquisas da Amazônia e Universidade Federal do Amazonas. Á CAPES pela concessão de bolsa de estudo ao autor sênior.

\section{REFERÊNCIAS}

Coscarón, S. \& Papavero, N. 1993. An illustrated manual for the identification of the Neotropical genera and subgenera of Tabanidae (Diptera). Museu Paraense Emílio Goeldi. Coleção Emilie Snethlage. $150 \mathrm{p}$.

Fairchild, G. B. 1964. Notes on Neotropical Tabanidae (Diptera). IV. Further new species and new records for Panama. Journal of Medical Entomology 1: 169-185.

Fairchild, G. B. 1975. Notes on Neotropical Tabanidae (Diptera). XVII. Genus Philipotabanus Fchld., subgenus Mimotabanus Fchld. Annals of the Entomologial Society of America 68: 689-694.

Fairchild, G. B. \& J. F. Burger. 1994. A catalog of the Tabanidae (Diptera) of the Americas South of the United States. Memoirs of the American Entomological Institute 55: 249 p.

Henriques, A. L. 2006. O gênero Philipotabanus Fairchild (Insecta: Diptera: Tabanidae) na Amazônia, com chave para as fêmeas e descrição de $P$. obidensis sp. nov. Acta Amazonica 36: 549-556.

McAlpine, J. F. 1981. Morphology and Terminology. pp. 9-63. In: J. F. McAlpine; B. V. Peterson; G. E. Shewell; H. J. Teskey; J. R. Vockeroth \& D. M. Wood (eds). Manual of Nearctic Diptera Vol 1. (Monograph $\mathrm{n}^{\circ}$ 27). Research Branch, Agriculture, 674 p.

Recebido em 10/11/2008; aceito em 04/06/2009 